\title{
Dynamic Balancing for Mixing Assembly Line Based on PSO - GA Cooperative Optimization
}

Yongming $\mathrm{Wu}^{1}$, Senquan $\mathrm{Lin}^{2}$, Longzhou Dai ${ }^{1}$, Lifei Luo ${ }^{1}$

${ }^{1}$ Modern Manufacturing Key Laboratory of Guizhou University, Guizhou Guiyang 550025. China. E-mail: wu20811055@163.com

${ }^{2}$ Zhangzhou Institute of Technology, Zhangzhou 363000. China. E-mail: kmlg2010@163.com

\begin{abstract}
Aiming at the evolution balance problem of hybrid assembly line (HAL), a dynamic (evolution) balancing model of assembly line is established, and a cooperative optimization algorithm (PSO-GA) is proposed in this paper. Firstly, HAL evolution in the market diversification and technology progress environment is studied, and assembly line evolution balance is mainly considered. In the PSO-GA, minimizing the number of stations, minimizing the load indexes of between stations and within each station, and adjusting costs are used as optimization objectives. For increasing population diversity and speeding up the searching speed, the individual information exchange and mutation are carried out in the populations. Finally, the effectiveness and feasibility of the method were proved by optimizing the HAL for an enterprise.
\end{abstract}

Keywords: Hybrid assembly line, Evolution, PSO-GA optimization, Evolution balancing

\section{Acknowledgments}

This work was supported by the National Natural Science Foundation of China (grant no. 51505094), the Guizhou Provincial Natural Science Foundation, China (no.[2016]1037), Applied basic research program of major projects in Guizhou(JZ[2014]2001) and talent introduction research program of Guizhou University(2014)60.

\section{References}

[1] GYÖRGY K. (2017). Productivity Improvement of Assembly Lines by Lean Methods. Manufacturing Technology, Vol.17, No.2, pp.192-197. Univerzita J. E., Purkyne.

[2] DARINA K., FILIP G., ANDRZEJ M., Damian G. (2013). Utilization of Advanced Simulation Methods for Solving of Assembly Processes Automation Partial Tasks. Manufacturing Technology, Vol.13, No.2, pp.478-486. Univerzita J. E., Purkyne.

[3] LIU Q., YAN Y.R., CAO L.L. (2016). Computer Visual Measurement Technology and Algorithm Simulation for the Assembly of Large Aircraft Parts. Manufacturing Technology, Vol. 16, No.3,pp.538-543. Univerzita J. E., Purkyne.

[4] YOU J.C., LIN J.D., XU D.F., HAO W.Y. (2016). Contact Analysis of Silicone Rubber Rectangular Ring in the Automatic Tighten Assembly. Manufacturing Technology, Vol. 16, No. 3, pp.648-653. Univerzita J. E., Purkyne.

[5] AKPINAR, S., BAYHAN, G. M., BAYKASOGLU, A. (2013). Hybridizing ant colony optimization via genetic algorithm for mixed-model assembly line balancing problem with sequence dependent setup times between tasks. Applied Soft Computing, Vol. 13, No.1, pp.574-589. Elsevier, Ltd.

[6] AKPINAR, S., BAYHAN, G. M. (2011). A hybrid genetic algorithm for mixed model assembly line balancing problem with parallel workstations and zoning constraints. Engineering Applications of Artificial Intelligence, Vol. 24, No.3, pp.449-457. Elsevier Ltd, United Kingdom. Elsevier, Ltd.

[7] BRYAN A, KO J, HU S J, KOREN, Y. (2007). Co-evolution of product families and assembly systems. CIRP Annals-Manufacturing Technology, Vol.56, No.1,pp. 41-44. Elsevier, USA.

[8] YANG C, GAO J, SUN L. (2013). A multi-objective genetic algorithm for mixed-model assembly line rebalancing. Computers \& Industrial Engineering, Vol.65, No.1, pp.109-116. Elsevier, Ltd.

[9] Zhu X, Hu SJ, Koren Y, Huang N.(2012). A complexity model for sequence planning in mixed-model assembly lines. Journal of Manufacturing Systems, Vol. 31,pp.121-130. Elsevier B.V. Netherlands.

[10] ZENG, X., WONG, W. K., LEUNG, Y. S. (2012). An operator allocation optimization model for balancing control of the hybrid assembly lines using pareto utility discrete differential evolution algorithm. Computers \& Operations Research, Vol. 39, No.5, pp.1145-1159. Elsevier, Ltd.

[11] UGUR O., BILAL T.(2009).Balancing of mixed-model two-sided assembly lines.Computers \& Industrial Engineering, Vol. 57,pp.217-227. Elsevier, Ltd. 
[12] NEDA M., MASOUD R., DAVOUD M., FARIBORZ J.(2012). Mixed-model assembly line balancing in the make-to-order and stochastic environment using multi-objective evolutionary algorithms.Expert Systems with Applications, Vol. 39,pp.12026-12031. Elsevier, Ltd.

[13] COROMINAS, A, PASTOR, R,PLANS, J.(2008).Balancing assembly line with skilled and unskilled workers.Omega, Vol. 36, No. 6,pp.1126-1132. Elsevier, Ltd.

[14] HOU L.,WU Y.M., LAI R.S., Tsai C.T.(2014).Product family assembly line balancing based on an improved genetic algorithm. Int J Adv Manuf Technol, Vol.70, pp.1775-1786. Springer, London.

Paper number: M2017112

Copyright (C) 2017. Published by Manufacturing Technology. All rights reserved. 